\title{
Blockage Detection in Circular Pipe Using Vibration Analysis
}

\author{
N. L. T. Lile ${ }^{1}$, M. H. M. Jaafar ${ }^{2}$, M. R. Roslan ${ }^{1}$, M. S. Muhamad Azmi ${ }^{1}$ \\ ${ }^{I}$ School of Mechatronic Engineering, Universiti Malaysia Perlis, Malaysia \\ E-mail: liyanatajul@unimap.edu.my,morafiq09@gmail.com,safwanazmi@unimap.edu.my \\ ${ }^{2}$ School of Manufacturing Engineering, Universiti Malaysia Perlis, Malaysia \\ E-mail: hasnulhadi@unimap.edu.my
}

\begin{abstract}
Pipe is an important medium used in most industrial and home applications for transferring liquid or gas from one end to the other. The efficiency of liquid flow is crucial to ensure proper and efficient delivering of medium carried. Liquid flow may be abrupt or ceased if blockage forms inside the circular pipe. This paper investigates the effect of blockage in circular pipe using vibration measurement. The main focus of this paper is to study the correlation of blockage levels to vibration signal. When fluid flow through an obstacle, the streamlines get closer which will increase the flow velocity and decrease the pressure. The vibration parameters are measured using accelerometer and the relationship between blockage levels to vibration signal are observed. It is found that vibration in pipe increases as the flow area gets smaller. This work expresses the potential of vibration analysis in assessing blockage inside a circular pipe with direct water flow.
\end{abstract}

Keywords - vibration; blockage; circular pipe; fluid flow.

\section{INTRODUCTION}

Pipe is one of the means used widely to transport or send liquid or gas from a place to another. Pipes can be found in houses, buildings, sewage and industries where the needs of sending fluid without any obstructions are crucial. Sometimes for example in a piping system the liquid is not flowing continuously or even stop flowing due to impurities clogging on the internal area of the pipe. This would cause inefficiency in terms of delivering time and also the volume rate being transferred.

In areas involving food processing, offshore pipelines and drainage system, where pipelines are very much in used, disturbance in pipe flows will create difficulties. Liquid with impurities and external objects entering the pipe will cause gradual built-up on the pipe surface area. Corrosion and rusting in pipes could also contribute to the irregularities in pipes where metal loss tends to deposit on the inner pipe surface. Apart from rusting, blockage precipitates inside pipes through supercooled water. Dendritic ice tends to form in water pipes under this condition since the nucleation of ice occurs between -4 to $-6^{\circ} \mathrm{C}[1]$.

In some applications, small built up is not considered as major setback, yet if this phenomenon is not taken into deliberation in early stage, the flow of fluid will curtail. In some cases clogging could cause the pipe to burst due to internal pressure change.
A number of researches have been conducted to identify blockage or obstacle in pipe to ensure the quality of medium being transferred. Reference [2] utilizes the rapid spectrum method to determine blockage in duct via single measurement of transfer function. Another investigation performed in [3] evaluates the effects of blockages on the nuclear power plant pressure transmitter sensing lines.

Noticing the consequences of clogging, this paper discusses the impact of blockage in circular pipe. There are many ways of detecting faults in pipes and one of them is by non-destructive testing (NDT). This work focuses on determining the relationship of blockage levels towards the vibration pattern signal where the signals are generated from vibrating body of pipe. The changes in the vibration parameters with the varying blockage size are observed.

\section{METHODOLOGY}

The specimen used in this work is circular carbon steel pipe. This steel pipe of length $1000 \mathrm{~mm}$ and diameter of 30 $\mathrm{mm}$ is shown in Fig. 1. At the centre of the pipe, a blockage block is attached to create flow-blocking situation (Fig. 2). There are a total of four different blockage thicknesses of $5 \mathrm{~mm}, 10 \mathrm{~mm}, 15 \mathrm{~mm}$ and $20 \mathrm{~mm}$ attached inside the pipe.

\section{A. Simulation}

Simulation using SolidWorks Flow Simulation was conducted to observe the flow pattern inside the pipe. Water flow rate was set to 20 LPM (liter per minute). Five different 
simulations were conducted to evaluate four different blockage thicknesses and a pipe with absence of blockage. Two flow parameters are taken into accounts which are flow velocity and pressure within the pipe.

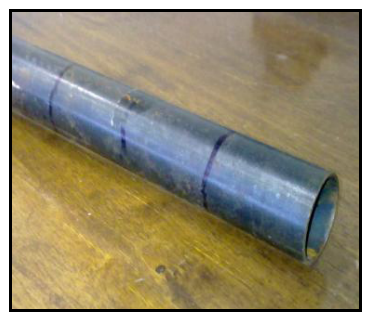

Fig. 1 Carbon Steel Pipe

\section{B. Experimental Work}

The experimental setup in Fig. 2 shows the circular pipe, blockage placement and the accelerometer mounting locations. The circular pipe is connected to water supply and placed horizontally. Water is allowed to flow through the pipe. Accelerometer with a reference sensitivity of $10.0 \mathrm{mV} / \mathrm{g}$ was utilised to obtain the vibrating response when water flows pass the irregularity.

Accelerometer was put on three different locations of $250 \mathrm{~mm}$ before blockage (Point A), on blockage (Point B) and $250 \mathrm{~mm}$ after blockage (Point $\mathrm{C}$ ). These different points of measurement will help in evaluating the vibration signal behaviour throughout the flow. The corresponding acceleration signal response is analysed by the LMS instrument.

\section{RESULT}

The pipe vibration response was studied based on different blockage sizes and different accelerometer locations. The response was observed based on the signal patterns, amplitude and frequency. Results are shown in two parts. First is the observation of vibration response towards different accelerometer locations while the second part is the blockage size monitoring.

\section{A. Simulation Result}

The streamlines of fluid flow in the pipe are shown in Fig. 3. Fig. 3(a) is the simulation result of pipe without blockage. The streamlines are distributed almost evenly within the inner pipe area. The streamlines of the velocity profile shows a uniform pattern. Based on [4], a fully developed laminar pipe flow occurs when there is no blockage in the system.

As the blockage is getting larger, the streamlines get closer and the gaps between the lines are narrower (Fig. 3(b)-(e)). These behaviors can be seen clearly when the water flow passes the blockage. The streamlines start to adjust as the fluid begins flowing through the obstacle. Water velocity inside the pipe increases as the flow area decreases.

The highest velocity was recorded for $20 \mathrm{~mm}$ blockage (Fig. 3(e)). The streamlines show that part of the flow is reversed towards the blockage forming recirculation behind the block. The flow inside the pipe becomes turbulent as blockage gets larger.

The pressure changes are calculated from the velocity profile and shown in Table 1. It can be seen that pressure during blockage drops as the restriction of flow increases. According to [5],[6] when fluid flow through an orifice, high flow velocity and small restriction in pipe will cause pressure to drop.

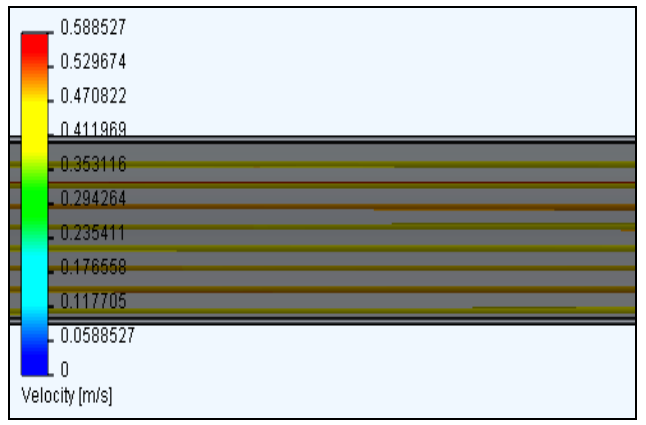

Fig. 3(a) No blockage

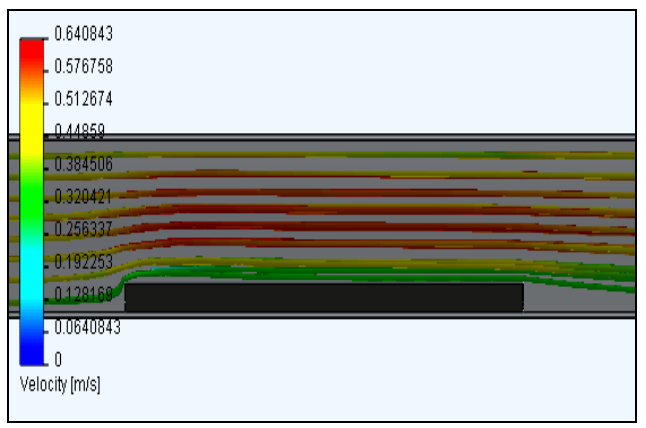

Fig. 3(b) $5 \mathrm{~mm}$ blockage

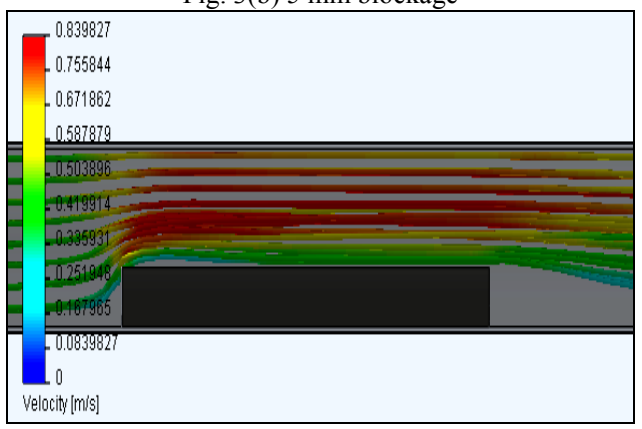

Fig. 3(c) $10 \mathrm{~mm}$ blockage

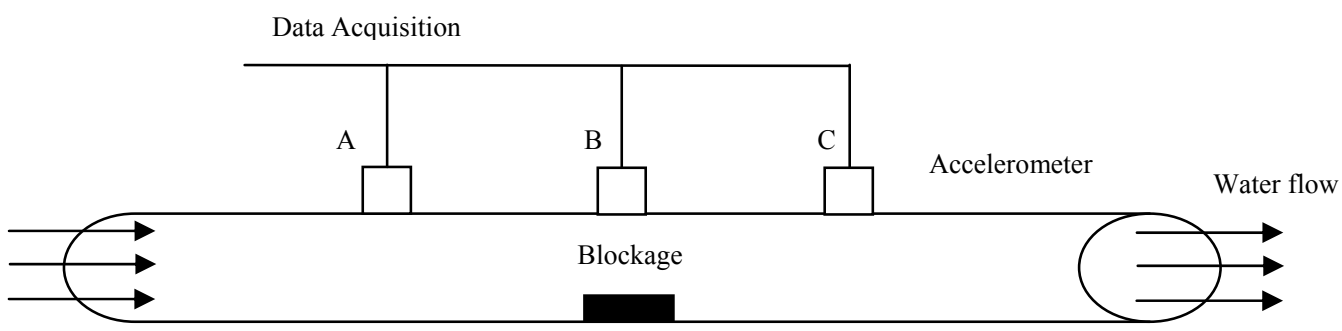

Fig. 2 Experimental Setup 


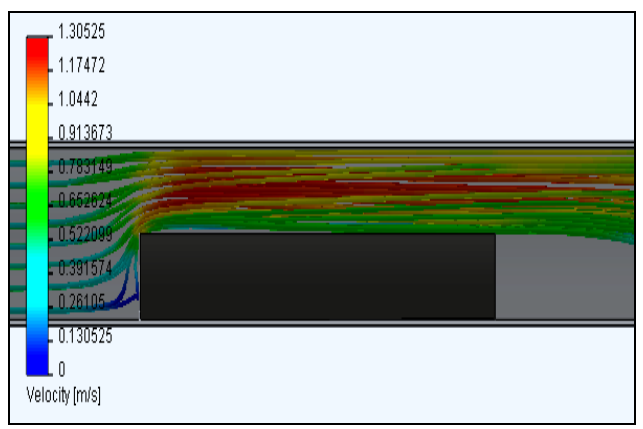

Fig. 3(d) $15 \mathrm{~mm}$ blockage

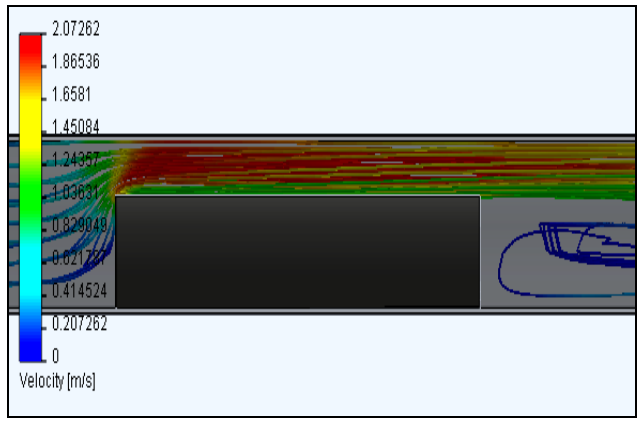

Fig. 3(e) $20 \mathrm{~mm}$ blockage

TABLE I

VELOCITY AND PRESSURE

\begin{tabular}{|l|l|l|}
\hline $\begin{array}{l}\text { Blockage } \\
\text { Thickness } \\
\text { (mm) }\end{array}$ & $\begin{array}{l}\text { Maximum } \\
\text { Fluid } \\
\text { Velocity } \\
\text { (m/s) }\end{array}$ & $\begin{array}{l}\text { Pressure at } \\
\text { Blockage } \\
\text { (Psi) }\end{array}$ \\
\hline 0 & 0.589 & 6.000 \\
\hline 5 & 0.638 & 5.997 \\
\hline 10 & 0.864 & 5.980 \\
\hline 15 & 1.349 & 5.925 \\
\hline 20 & 2.198 & 5.771 \\
\hline
\end{tabular}

\section{B. Experimental Result}

Vibration signal pattern and parameters were observed throughout this work. The vibration acceleration response is measured in $\mathrm{m} / \mathrm{s}^{2}$. Two types of signal are observed through the data acquisition system analyser which are Fast Fourier Transform (FFT) and Root Mean Square (RMS) of the vibration acceleration response.

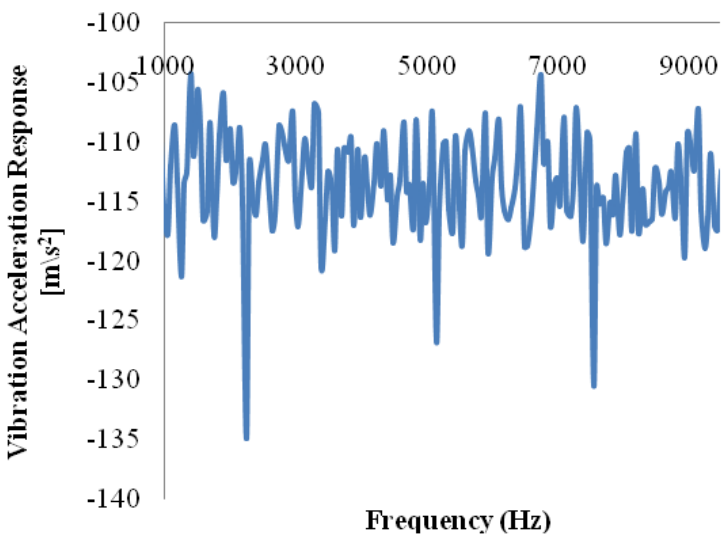

Fig. 4 Vibration acceleration response (Point A) of $5 \mathrm{~mm}$ blockage thickness
Fig. 4 depicts the FFT graph of vibration acceleration response. The FFT graph shows the relationship of acceleration and frequency taken when water flow through a blockage of thickness $5 \mathrm{~mm}$ at Point A $(250 \mathrm{~mm}$ before blockage).

Fig. 5 is the plot of frequency for different blockage thicknesses and different accelerometer locations of Point A, $\mathrm{B}$ and $\mathrm{C}$. The frequency shows a clear correlation between blockage levels and accelerometer locations. The plot of Point A shows almost constant or no significant changes in frequency. Since Point A is $250 \mathrm{~mm}$ before blockage occurred, the cross sectional area inside the pipe has not undergo any changes. Therefore the streamlines and the pressure inside the pipe at this point do not vary.

Comparing the frequencies of Point A, B and C, frequencies of Point $\mathrm{B}$ are the highest since the acceleration responses are taken on the pipe where blockage occurred. This is the area where vibration response is the highest due to fluctuating pressure and high velocity of fluid. The changes in the water velocity and also pressure inside the pipe will increase the vibration of the circular pipe.

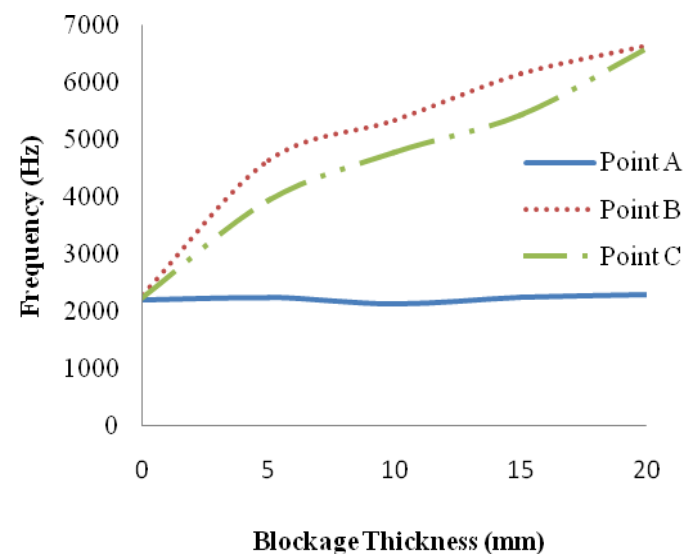

Fig. 5 Frequency response of different accelerometer locations

The RMS signal of different blockage thicknesses is shown in Fig. 6. The RMS signal is measured on the point of blockage. The graph shows that the RMS values increase with blockage thickness. In a constant water flow, larger blockage in pipe will create higher vibration. According to [7], small hole orifice inside a pipe will cause greater vibration. Blockage here will reduce the flow area of fluid inside a pipe. Therefore flow area will get smaller while vibration amplitude becomes greater.

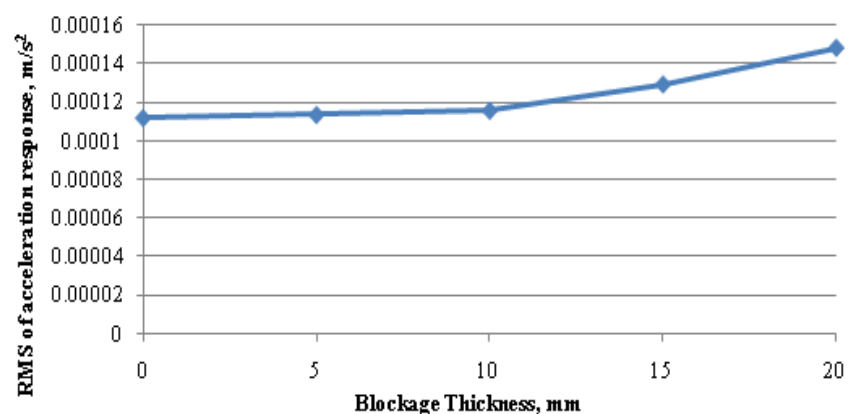

Fig. 6 RMS response at different blockage thicknesses 


\section{IV.CONCLUSION}

This paper presents a non-destructive testing method on evaluating blockage in pipe. Simulation analysis was conducted to observe the effect of blockage size on the flow streamlines. When water flow passes the small restriction, velocity will increases and pressure will drop. Vibration measurement has been conducted to discover the correlation between blockage size and vibration signal. In a pipe, location with greater obstruction will cause higher vibration.

\section{ACKNOWLEDGMENT}

This work was financially supported by the Ministry of Higher Education (MOHE), Malaysia under the Fundamental Research Grant Scheme (FRGS).

\section{REFERENCES}

[1] R. R. Gilpin, "The effects of dendritic ice formation in water pipes," in Int. J. Heat Mass Transfer, 1977, vol. 20, pp. 693-699.

[2] M. H. F De Salis and D. J. Oldham, "The development of a rapid single spectrum method for determining the blockage characteristics of a finite length duct," in Journal of Sound and Vibration, 2001, 243(4), pp. 625-640

[3] K. Lin and K. E. Holbert, "Blockage diagnostics for nuclear power plant pressure transmitter sensing lines," in Nuclear Engineering and Design, 2009, vol. 239, pp. 365-372.

[4] M. Toophanpour-Rami, E.R. Hassan, R. M. Kelso and J.P. Denier, "Preliminary investigation of impulsively blocked pipe flow", in 16th Australasian Fluid Mechanics Conference (AFMC), 2007, pp. 967970 .

[5] Q. Mao, Y. Zhang, W. Xiang, Q. Bi, H. Li, F. Zang and Q. Duan, "High-level vibration and noise analysis of nuclear pipes with orifice," in Transactions of 17th International Conference on Structural Mechanics in Reactor Technology (SMiRT 17), 2003.

[6] Y. Kim, A. R. Simpson, and M. F. Lambert, "The effect of orifices and blockages on unsteady pipe flows", in World Environmental and Water Resources Congress, 2007.

[7] Q. Mao and J. Zhang, "Orifice-induced wall pressure fluctuations and pipe vibrations: theory and modeling of fluid excitations", in Flow, Turbulence and Combustion, 2007, vol. 79, pp. 25-40. 